\title{
Fotossíntese de espécies de Anibae em resposta à exposição a ambientes contrastantes de luz
}

Photosynthetic responses of species of Anibae exposed to contrasting light environments

\author{
Michell Richard Blind ${ }^{1}$, Karen Cristina Pires da Costa $^{1}$, Carlos Eduardo Moura da Silva ${ }^{2}$, \\ Paulo de Tarso Barbosa Sampaio ${ }^{3} \&$ José Francisco de Carvalho Gonçalves ${ }^{1,4}$
}

\begin{abstract}
Resumo
Fotossíntese de espécies de Anibae em resposta à exposição a ambientes contrastantes de luz. Características ecofisiológicas e o ambiente de luz têm implicações sobre o estabelecimento de plantios florestais. O objetivo desse estudo foi investigar a fotossíntese de Aniba canelilla e A. rosaeodora em resposta à disponibilidade de luz no campo. As trocas gasosas, conteúdo de clorofilas (ICC) e desempenho fotoquímico $\left(\mathrm{F}_{\mathrm{V}} / \mathrm{F}_{\mathrm{M}}\right)$ foram medidos em folhas aclimatadas (sombra durante 300 dias), a pleno sol (28 dias) e novamente à sombra durante 28 dias. A fotossíntese, $\mathrm{ICC}$ e $\mathrm{F}_{\mathrm{V}} / \mathrm{F}_{\mathrm{M}}$ de $A$. canelilla e $A$. rosaeodora foram afetadas negativamente a pleno sol, mas se recuperaram quando submetidas à sombra. A fotossíntese, ICC e $\mathrm{F}_{\mathrm{V}} / \mathrm{F}_{\mathrm{M}}$ foram duas vezes superiores nas plantas de sombra. Aniba rosaeodora apresentou desempenho fotossintético duas vezes superior a $A$. canelilla. As diferenças nas respostas fotossintéticas à luz sugerem que as espécies poderiam ocupar "nichos" de sucessão diferentes em plantios de enriquecimento e, a plasticidade (mediada pela recuperação) implica aclimatação em ambientes de luz variável. O fato das espécies de Anibae estudadas possuírem diferenças de plasticidade para luz deve ser visto com cautela para sugerir plantios convencionais, mas reforça a indicação para plantios de enriquecimento em sítios florestais com variações na disponibilidade de luz.

Palavras-chave: Aniba canelilla, Aniba rosaeodora, fluorescência da clorofila a, plantios florestais, trocas gasosas.
\end{abstract}

\begin{abstract}
Photosynthesis of species of Anibae in response to the exposure to contrasting light environments. Ecophysiological traits and the light environment have implications for the establishment of forest plantations. The aim of this study was to investigate the photosynthetic activity of Aniba canelilla and A. rosaeodora in response to changes in the availability of light in the field. Gas exchange, content of chlorophylls (ICC) and photochemical performance $\left(\mathrm{F}_{\mathrm{V}} / \mathrm{F}_{\mathrm{M}}\right)$ were measured in leaves acclimated in the shade during 300 days, after in the full sun (28 days) and again in the shade by 28 days. The photosynthesis, ICC and $\mathrm{F}_{\mathrm{V}} / \mathrm{F}_{\mathrm{M}}$ of $A$. canelilla and $A$. rosaeodora were negatively affected in full sunlight, but the plants recovered when submitted to shade. Photosynthesis, ICC and $\mathrm{F}_{\mathrm{V}} / \mathrm{F}_{\mathrm{M}}$ were twice as high in shade plants. Aniba rosaeodora presented photosynthetic performance twice higher than $A$. canelilla. Differences in photosynthetic responses to light suggest that species could occupy different succession "niches" in enrichment plantation, and plasticity (recovery-mediated) implies acclimatization under variable light environments. The fact that the studied species of Anibae have differences in plasticity for light should be viewed with caution to suggest conventional plantations, but it reinforce the indication for enrichment plantations in forest sites with variations in the availability of light.
\end{abstract} Key words: Aniba canelilla, Aniba rosaeodora, chlorophyll $a$ fluorescence, plantation, gas exchange.

\footnotetext{
${ }^{1}$ Instituto Nacional de Pesquisas da Amazônia, Coord. Dinâmica Ambiental, Lab. Fisiologia e Bioquímica Vegetal, Av. Efigênio Sales 2239, Aleixo, 69066-970, C.P. 476, Manaus, AM, Brasil.

${ }^{2}$ Universidade Estadual de Roraima, Av. Senador Helio Campus s/n, Rorainópolis, RR, Brasil.

${ }^{3}$ Instituto Nacional de Pesquisas da Amazônia, Coord. Tecnologia e Inovação, Lab. Propagação de Plantas, Av. Efigênio Sales 2239, Aleixo, 69066-970, C.P. 476, Manaus, AM, Brasil.

${ }^{4}$ Autor para correspondência: jfc@inpa.gov.br
} 


\section{Introdução}

As espécies florestais casca preciosa (Aniba canelilla (Kunth) Mez) e pau rosa (A. rosaeodora Ducke) pertencentes à família Lauraceae estão entre as mais valiosas árvores do Bioma Amazônia. Ambas produzem óleos essenciais altamente valorizados pelas indústrias de perfumaria e farmacêutica (Amusant et al. 2016; Giongo et al. 2017). O alto valor comercial dos óleos contribui para a superexploração de populações nativas dessas espécies, o que resultou na inserção de A. rosaeodora na lista de espécies ameaçadas de extinção (International Union for Conservation of Nature 2017).

Do ponto de vista da ecologia das espécies, em particular, da sucessão florestal, as espécies pertencentes à família Lauraceae são classificadas como espécies secundárias ou sucessionais tardias (Silva et al. 2003; Zama et al. 2012). Estas evidências são semelhantes para Aniba canelilla e $A$. rosaeodora que têm sido classificadas como espécies clímax tolerantes à sombra (Sampaio et al. 2003; Karsten et al. 2013). Embora, tenha sido verificado que a regeneração dessas espécies seja, em algumas situações, dependente da abertura de clareiras com maior incidência de luz (Sampaio et al. 2003; Pinheiro et al. 2007; Karsten et al. 2013). Diante disto, percebe-se a relevância das características ecofuncionais destas espécies, isto é, como o fator do meio (ex.: ambiente lumínico) propicia alterações no comportamento fisiológico destas plantas e também como suscitam eventuais controvérsias quanto a real posição sucessional das mesmas. Estas inferências "per si" sugerem a necessidade de maior aprofundamento neste assunto visando à aplicação destes conhecimentos no que se refere aos plantios e ao manejo destas espécies na Amazônia.

O elevado valor econômico das espécies da família Lauraceae, sobrepõe os entraves da baixa taxa de regeneração natural e têm sido estimuladas iniciativas de plantios dessas espécies na região Amazônica, em especial de Aniba rosaeodora (Amusant et al. 2015). Entretanto, outras dificuldades relacionadas à obtenção de sementes e ao manejo das plantações têm comprometido o crescimento desses empreendimentos (Sampaio et al. 2003; Amusant et al. 2015).

Quanto ao estabelecimento dos plantios de espécies arbóreas nativas, de maneira geral, as informações limitadas e/ou inconsistentes relacionadas às respostas ecofisiológicas destas espécies quanto à disponibilidade de recursos primários constitui fator limitante para a seleção de espécies e para estimular o aumento das áreas de plantios florestais (Gonçalves et al. 2005; Ribeiro et al. 2005; Goldstein et al. 2016). Essas informações são fundamentais para planejar diferentes sistemas de plantios, sejam aqueles voltados para produção florestal, sistemas agroflorestais ou mesmo plantios de enriquecimento em florestas secundárias (Tanaka \& Vieira 2006). Levantamento de informações de uso e cobertura da terra na Amazônia, em 2012, indica que as florestas secundárias representam $22 \%$ das áreas desflorestadas da Amazônia Legal (Instituto Nacional de Pesquisas Espaciais 2017) e que estas coberturas florestais, mesmo de forma limitada, desempenham importantes serviços ecológicos, no que concerne ao início da nucleação, abrigo para dispersores de sementes e o sequestro de carbono atmosférico (Ferraz et al. 2014; Wandelli \& Fearnside 2015). Porém, estas áreas são pobres em diversidade arbórea onde se verifica a predominância de espécies de baixo valor comercial, como as pertencentes aos gêneros Vismia, Bellucia, Laetia e Goupia (Silva et al. 2008; Reis et al. 2014). Deste modo, o enriquecimento de florestas secundárias, como sistema silvicultural, representa uma alternativa para a reintrodução dessas áreas aos processos produtivos, além de poder reduzir a pressão de exploração sobre as florestas primárias remanescentes (Rappaport \& Montagnini 2014; Doucet et al. 2016).

Do ponto de vista técnico, o método de plantio mais utilizado nos sistemas de enriquecimento é o de linhas ou faixas, mas em áreas de florestas manejadas, o plantio em clareiras tem sido mais aplicado (Reis et al. 2014). Nos primeiros anos de plantio, há uma maior disponibilidade de luz direta, que pode ser decisivo para o estabelecimento das plantas no campo devido à intensificação das taxas de transpiração e aos eventos de fotoinibição (Tanaka \& Vieira 2006). Após este período, quando a vegetação adjacente começa a crescer, as plantas são sombreadas, no caso de espécies exigentes em luz, isso pode resultar em redução das taxas fotossintéticas e, por conseguinte, do crescimento, mas por outro lado, também pode favorecer aquelas espécies que exigem menor quantidade de luz, criando "nichos" ou ambientes lumínicos que podem ser estabelecidos gradativamente. Deste modo, a seleção de espécies capazes de se aclimatar à variação na disponibilidade de irradiância que naturalmente ocorre em plantios de enriquecimento de florestas secundárias pode representar importante técnica alternativa, menos 
onerosa e de baixo impacto com potencial sucesso destas iniciativas em florestas tropicais.

Na literatura já existem evidências que Aniba canelilla e $A$. rosaeodora, quando submetidas à alta irradiância, desenvolvem estratégias de fotoproteção, porém diminuem o desempenho fotossintético (Gonçalves et al. 2005; Atroch 2008). No entanto, não há informações consistentes de como essas espécies se comportam diante da variação na disponibilidade de luz e se seriam capazes de se recuperar após o período de estresse por alta irradiância. Nós examinamos neste artigo a hipótese que a eventual plasticidade diferencial à luz das espécies de Anibae pode ser utilizada a favor do adequado e gradativo plantio destas espécies em sistemas de enriquecimento de clareiras na Amazônia.

No presente estudo foi investigado o desempenho fotossintético de plantas jovens de Aniba canelilla e $A$. rosaeodora expostas a condições contrastantes de irradiância no campo. O objetivo foi responder as seguintes questões: $\left(1^{\circ}\right)$ Quais os efeitos da variação na disponibilidade de luz sobre o desempenho fotossintético de $A$. canelilla e $A$. rosaeodora? e ( $\left.2^{\circ}\right)$ Qual o nível de plasticidade fisiológica destas espécies de Anibae quando submetidas a condições contrastantes de irradiância (sombra e pleno sol)?

\section{Material e Métodos}

\section{Área de estudo}

O estudo foi realizado no Sítio São Gonçalo (1 ${ }^{\circ} 56^{\prime} 00^{\prime}$ 'S e 6001'45'W), município de Presidente Figueiredo - Amazonas. O clima é do tipo Af, ou seja, tropical úmido (Köppen 1948), com temperatura e precipitação anual média de $27^{\circ} \mathrm{C}$ e $2.500 \mathrm{~mm}$, respectivamente (Instituto Nacional de Meteorologia 2017). A área foi utilizada para plantios agrícolas até 2007 e em seguida foi abandonada.

\section{Produção de mudas}

As mudas de Aniba rosaeodora e A. canelilla foram produzidas durante quatro meses no viveiro (telado por sombrite 50\%) da Estação de Silvicultura Tropical da Universidade do Estado do Amazonas (EST/UEA). As sementes foram coletadas em árvores matrizes da Reserva Florestal Adolpho Ducke (Rodovia Am-010, km 26 - Manaus/AM).

O plantio

O plantio foi realizado em terreno com topografia plana e em solo classificado como
Podzólicos Vermelho Álico, segundo descrição de Nava et al. (1998). Na ocasião do plantio, as mudas de Aniba rosaeodora possuíam $35 \pm 5 \mathrm{~cm}$ de altura e cerca de $8 \pm 5$ folhas, enquanto $A$. canelilla apresentava altura média de $38 \pm 6 \mathrm{~cm}$ e $14 \pm 6$ folhas. $\mathrm{O}$ espaçamento utilizado foi de $1 \times 1 \mathrm{~m}$. O tamanho das covas foi de $30 \times 30 \mathrm{~cm}$ de largura e profundidade e as covas receberam calcário 20 dias antes do plantio.

\section{Experimento}

O plantio das mudas foi realizado sob cobertura de tela sombrite $50 \%$. O sombrite foi colocado em uma estrutura de palanques a $1 \mathrm{~m}$ de altura do solo. As plantas permaneceram nesta condição durante 300 dias. Em seguida, foram expostas a pleno sol durante 28 dias. Após 28 dias, o sombrite foi novamente colocado sobre as plantas e assim permaneceram por mais 28 dias.

A irradiância sobre as plantas durante o período de aclimatação foi de $200 \mu \mathrm{mol}$ (photon) $\mathrm{m}^{-2} \mathrm{~s}^{-1}$, quando as plantas foram expostas a pleno sol a irradiância aumentou para 1.500 (photon) $\mu \mathrm{mol}$ $\mathrm{m}^{-2} \mathrm{~s}^{-1}$, e quando submetidas ao sombreamento novamente, a irradiância caiu para 300 (photon) $\mu \mathrm{mol} \mathrm{m} \mathrm{m}^{-2} \mathrm{~s}^{-1}$. A irradiância foi medida entre 8:00 e 11:30 h durante quatro dias em cada ambiente de luz, para tanto utilizou-se um sensor quântico (Li190 SA, Li-Cor, EUA) conectado a um datalogger.

Os dados de trocas gasosas, conteúdo de clorofilas e a fluorescência da clorofila $a$ foram coletados no $300^{\circ}$ dia de aclimatação e durante os períodos em que as plantas foram expostas a pleno sol e ao sombreamento.

\section{Trocas gasosas}

As taxas de fotossíntese $(A)$, condutância estomática $\left(g_{s}\right)$, transpiração $(E)$, respiração no escuro $\left(R_{d}\right)$ e concentração interna de $\mathrm{CO}_{2}(\mathrm{C} i)$ foram medidas com um analisador de gás a infravermelho (IRGA) portátil, de sistema aberto, modelo LI-6400 (Li-Cor, USA) equipado com fonte de luz artificial 6400-02B Red Blue. As determinações foram realizadas em folhas em bom estado fitossanitário e completamente expandidas, localizadas no terço médio das plantas.

Os valores pontuais de trocas gasosas foram obtidos à densidade de fluxo de fótons saturante (PPFD) de $1.000 \mu \mathrm{mol} \mathrm{m} \mathrm{m}^{-2} \mathrm{~s}^{-1}$. O IRGA foi ajustado para concentração de $\mathrm{CO}_{2}$ e $\mathrm{H}_{2} \mathrm{O}$ dentro da câmara de medição em torno de $380 \pm 5 \mu \mathrm{mol} \mathrm{mol}^{-1}$ e 21 $\pm 1 \mathrm{mmol} \mathrm{mol}^{-1}$, respectivamente, e a temperatura do bloco foi mantida em torno de $30{ }^{\circ} \mathrm{C} \pm 1{ }^{\circ} \mathrm{C}$. (Silva et al. 2008; Silva et al. 2011). 
Conteúdo de clorofilas

O índice de conteúdo de clorofilas (ICC) foi determinado nas mesmas folhas utilizadas para as leituras das trocas gasosas. Para tanto, utilizou-se um clorofilômetro portátil (modelo CCM-200, OptiScience) (Gonçalves et al. 2008; Parry et al. 2014).

\section{Fluorescência da clorofila a}

A fluorescência da clorofila $a$ foi determinada utilizando-se um fluorômetro portátil (PEA, MK2 - 9600 - Hansatech, Norfolk, UK). Os dados foram coletados entre 8 e $12 \mathrm{~h}$. As medições foram realizadas nas mesmas folhas utilizadas para a determinação das trocas gasosas. As folhas foram inicialmente aclimatadas ao escuro durante 30 minutos, em seguida foram submetidas a um pulso de luz saturante de intensidade de $3.000 \mu \mathrm{mol} \mathrm{m}^{-2} \mathrm{~s}^{-1}$ e comprimento de onda de $650 \mathrm{~nm}$ por 1 segundo (Gonçalves et al. 2005). As respostas relacionadas aos transientes da fluorescência da clorofila $a$ foram obtidas a partir de software específico (Handy PEA software - v 1,30) de acordo com as equações do teste JIP (Strasser et al. 1995).

\section{Plasticidade}

$\mathrm{O}$ índice de plasticidade (IP) foi calculado a partir da diferença entre o valor máximo e mínimo nos diferentes tratamentos de irradiância, divididos pelo valor máximo (Valladares et al. 2000).

\section{Delineamento experimental \\ e análises estatísticas}

O delineamento experimental utilizado foi o inteiramente casualizado. As análises foram realizadas considerando duas abordagens. Primeiramente, foram realizadas comparações entre as espécies. Para tanto, os dados foram submetidos ao teste de normalidade (Shapiro Wilks) e homogeneidade de variância ( $F$ máximo) e em seguida ao teste $t$ para amostras independentes, com dois tratamentos (espécies) e dez repetições (planta). Na segunda abordagem, as comparações foram realizadas entre as diferentes condições de luz (exposição a pleno sol e à sombra) para cada espécie. Os dados foram submetidos ao teste de normalidade e em seguida ao teste t pareado, com dois tratamentos (condições de luz) e dez repetições. Para as variáveis em que os dados não atenderam os pressupostos de normalidade e variâncias homogêneas, utilizou-se o teste de comparação de Wilcoxon. Todas as análises estatísticas foram processadas no software R versão 3.1.1.

\section{Resultados}

As características fotossintéticas de Aniba canelilla e A. rosaeodora foram influenciadas pela variação na disponibilidade de irradiância (Tab. 1; Fig. 1a-d). Para ambas as espécies observou-se que as taxas de fotossíntese $(A)$ reduziram quando as mudas foram expostas ao pleno sol e aumentaram quando submetidas novamente à sombra (Tab. 1). A condutância estomática $(g s)$ não foi alterada pela exposição das espécies ao pleno sol, porém aumentou quando submetidas novamente à sombra, ao passo que a concentração interna de $\mathrm{CO}_{2}(\mathrm{Ci})$ aumentou em pleno sol e reduziu sob a condição de sombra (Tab. 1).

Aniba canelilla e A. rosaeodora, sob sombra, alcançaram taxas de fotossíntese e condutância estomática duas vezes maiores à condição de pleno sol (Fig. 1a,b). Independente da condição de luz, o desempenho fotossintético de $A$. rosaeodora foi cerca de duas vezes superior à $A$. canelilla (Fig. 1a). Os valores de fotossíntese foram positivamente correlacionados com os valores da razão $\mathrm{F}_{\mathrm{V}} / \mathrm{F}_{\mathrm{M}}(p$ $=0,001, \mathrm{r}=0,78)$ (Fig. 2).

A variação na disponibilidade de luz influenciou o conteúdo foliar de pigmentos cloroplastídicos de Aniba canelilla e A. rosaeodora (Tab. 2). O índice de conteúdo de clorofilas (ICC) reduziu cerca de $5 \%$ em $A$. canelilla e $10 \%$ em $A$. rosaeodora quando expostas à condição de pleno sol. Por outro lado, sob sombra, os valores aumentaram nas ordens de 5 e $14 \%$, respectivamente (Tab. 2). O conteúdo foliar de pigmentos cloroplastídicos (ICC) de $A$. rosaeodora foi 53\% maior ao observado em A. canelilla (Tab. 2).

O desempenho fotoquímico de Aniba canelilla e A. rosaeodora também foi influenciado pela variação na disponibilidade de luz (Tab. 2; Fig. 3a-f). As eficiências na captação da energia $\left(\mathrm{TR}_{\mathrm{O}} /\right.$ $\mathrm{CS})$ e no transporte de elétrons $\left(\mathrm{ET}_{\mathrm{O}} / \mathrm{CS}\right)$ reduziram quando as plantas foram expostas ao pleno sol e aumentaram sob sombra (Tab. 2). Por outro lado, a dissipação não fotoquímica $\left(\mathrm{DI}_{\mathrm{O}} / \mathrm{CS}\right)$ apresentou comportamento inverso aos parâmetros $\mathrm{TR}_{\mathrm{O}} / \mathrm{CS}$ e $\mathrm{ET}_{\mathrm{O}} / \mathrm{CS}$ (Tab. 2). Em síntese, os valores de $\mathrm{TR}_{\mathrm{O}} / \mathrm{CS}$ e $\mathrm{ET}_{\mathrm{O}} / \mathrm{CS}$ sob sombra foram aproximadamente 2 vezes superiores aos observados em pleno sol para as duas espécies (Tab. 2).

A máxima eficiência fotoquímica do PSII $\left(\mathrm{F}_{\mathrm{V}} / \mathrm{F}_{\mathrm{M}}\right)$ e o índice de desempenho $\left(\mathrm{PI}_{\mathrm{ABS}}\right)$ também reduziram em pleno sol indicando ocorrência de fotoinibição. Porém, quando as plantas foram submetidas à sombra, os valores de $\mathrm{F}_{\mathrm{V}} / \mathrm{F}_{\mathrm{M}}$ e $\mathrm{PI}_{\mathrm{ABS}}$ 

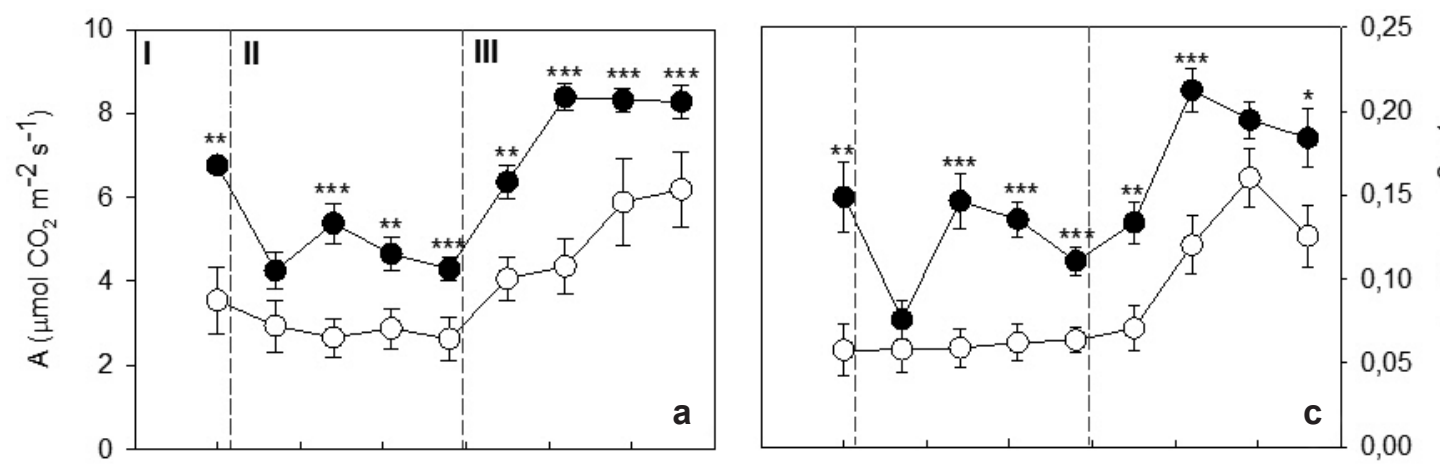

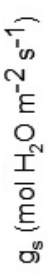

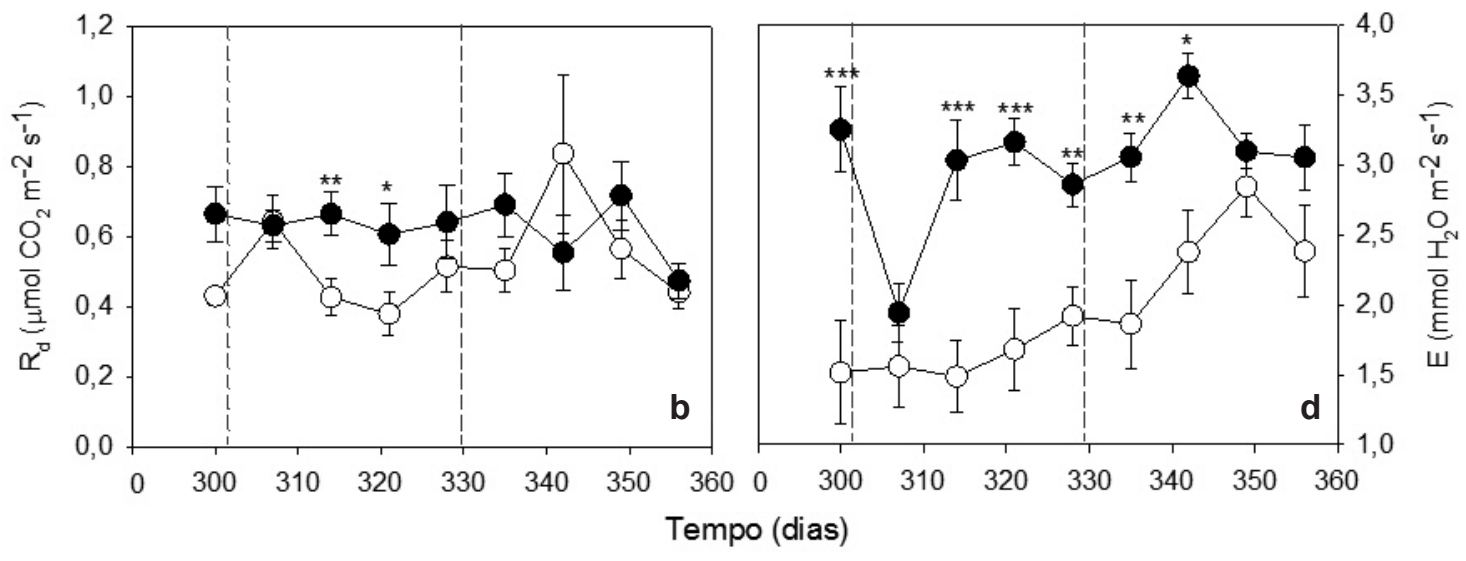

Figura 1 - Mudanças nas trocas gasosas de Aniba canelilla $(\bigcirc)$ e A. rosaeodora $(\bullet)$ promovidas pela variação na disponibilidade de luz $(\mathrm{n}=10)$. As linhas pontilhadas indicam o momento em que foi realizada a intervenção no plantio. I = aclimatação; II = pleno sol; III = sombreamento. a. fotossíntese $(A)$; b. respiração no escuro $\left(R_{d}\right)$; c. condutância estomática $\left(g_{s}\right)$; d. transpiração $(E)$.

Figure 1 - Changes in gas exchange of Aniba canelilla $(O)$ and A. rosaeodora $(\bullet)$ promoted by the variation in the availability of light $(\mathrm{n}=10)$. Dotted lines indicate the time it was conducted intervention in the planting. $\mathrm{I}=$ acclimatization; $\mathrm{II}=$ full sunlight; III $=$ shading. a. photosynthesis $(A)$; b. dark respiration $\left(R_{d}\right)$; c. stomatal conductance $\left(g_{s}\right)$; d. transpiration $(E)$.

Tabela 1 - Trocas gasosas e características fotossintéticas de plantas jovens de Aniba canelilla e A. rosaeodora submetidas à condições contrastantes de irradiância. Fotossíntese $(A)$, condutância estomática $\left(g_{s}\right)$, respiração no escuro $\left(R_{d}\right)$, transpiração $(E)$ e concentração interna de $\mathrm{CO}_{2}(C i)$.

Table 1 - Gas exchange and photosynthetic characteristics of young Aniba. canelilla and A. rosaeodora plants submitted to contrasting irradiance conditions. Photosynthesis $(A)$, stomatal conductance $\left(g_{s}\right)$, dark respiration $\left(R_{d}\right)$, transpiration $(E)$ and internal $\mathrm{CO}_{2}$ concentration $(\mathrm{Ci})$.

\begin{tabular}{|c|c|c|c|c|}
\hline \multirow{2}{*}{ Parâmetro } & \multicolumn{2}{|c|}{ Aniba canelilla } & \multicolumn{2}{|c|}{ Aniba rosaeodora } \\
\hline & Aclimatada - Sol & Sol - Sombra & Aclimatada - Sol & Sol - Sombra \\
\hline$A\left(\mu \mathrm{mol} \mathrm{CO}{ }_{2} \mathrm{~m}^{-2} \mathrm{~s}^{-1}\right)$ & $\mathbf{3 , 5 5}(-26)^{*}$ & $\mathbf{2 , 6 3}(+135)^{* * *}$ & $\mathbf{6 , 7 6}(-36)^{* *}$ & $\mathbf{4 , 3 0}(+92)^{* * *}$ \\
\hline 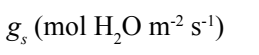 & $\mathbf{0 , 0 6}(+10)$ & $\mathbf{0 , 0 6}(+97)^{* *}$ & $0,15(-25)$ & $\mathbf{0 , 1 1}(+65)^{* *}$ \\
\hline$R_{d}\left(\mu \mathrm{mol} \mathrm{CO} \mathrm{m}^{-2} \mathrm{~s}^{-1}\right)$ & $\mathbf{0 , 4 3}(+20)$ & $\mathbf{0 , 5 1}(-15)$ & $0,66(-3)^{*}$ & $\mathbf{0 , 6 4}(-26)$ \\
\hline$E\left(\mathrm{mmol} \mathrm{H}_{2} \mathrm{O} \mathrm{m}^{-2} \mathrm{~s}^{-1}\right)$ & $\mathbf{1 , 5 2}(+27)$ & $1,92(+24)$ & $\mathbf{3 , 2 5}(-11)$ & $2,88(+6)$ \\
\hline$C i\left(\mu \mathrm{mol} \mathrm{CO} 2 \mathrm{~m}^{-2} \mathrm{~s}^{-1}\right)$ & $\mathbf{2 5 9 , 5 0}(+13)^{* *}$ & $293,43(-14)^{*}$ & $263,42(+11)^{* *}$ & $291,15(-7)^{* *}$ \\
\hline
\end{tabular}

Média de 10 repetições por período. As porcentagens entre parêntesis referem-se às mudanças promovidas pela condição de luz, valores negativos indicam redução em relação à condição de exposição anterior, enquanto valores positivos indicam aumento. * $\mathrm{p}<0,05 ; * * \mathrm{p}<0,01 ; * * * \mathrm{p}<0,001$. 
Tabela 2 - Índice de conteúdo de clorofilas e fluorescência da clorofila a de plantas jovens de Aniba canelilla e A. rosaeodora submetidas à condições contrastantes de irradiância. Índice do conteúdo de clorofilas (ICC), eficiência de absorção (ABS/CS), dissipação (DI $/ \mathrm{CS})$, aprisionamento $\left(\mathrm{TR}_{\mathrm{O}} / \mathrm{CS}\right)$ e transferência $\left(\mathrm{ET}_{\mathrm{O}} / \mathrm{CS}\right)$ de energia, rendimento quântico máximo do PSII $\left(\mathrm{F}_{\mathrm{V}} / \mathrm{F}_{\mathrm{M}}\right)$ e índice de desempenho na base $\mathrm{ABS}\left(\mathrm{PI}_{\mathrm{ABS}}\right)$.

Table 2 - Chlorophyll content and chlorophyll a fluorescence of young A. canelilla and A. rosaeodora plants submitted to contrasting irradiance conditions. Chlorophyll content (ICC), absorption flux per CS (ABS/CS), energy dissipation flux ( $\left.\mathrm{DI}_{\mathrm{o}} / \mathrm{CS}\right)$, exciton trapping flux $\left(\mathrm{TR}_{\mathrm{O}} / \mathrm{CS}\right)$ and electron transport flux $\left(\mathrm{ET}_{\mathrm{O}} / \mathrm{CS}\right)$, maximum quantum yield of $\mathrm{PSII}\left(\mathrm{F}_{\mathrm{V}} / \mathrm{F}_{\mathrm{M}}\right)$ and performance index based on $\mathrm{ABS}\left(\mathrm{PI}_{\mathrm{ABS}}\right)$.

\begin{tabular}{lcccc}
\hline \multirow{2}{*}{ Parâmetro } & \multicolumn{2}{c}{ Aniba canelilla } & \multicolumn{2}{c}{ Aniba rosaeodora } \\
\hline $\mathrm{ICC}$ & Aclimatada - Sol & Sol - Sombra & Aclimatada - Sol & Sol - Sombra \\
$\mathrm{ABS} / \mathrm{CS}$ & $\mathbf{2 5}(-5)^{* *}$ & $\mathbf{1 4}(+5)^{* *}$ & $\mathbf{1 7 , 1}(-10)^{* *}$ & $\mathbf{1 2}(+14)^{* * *}$ \\
$\mathrm{DI} / \mathrm{CS}$ & $\mathbf{9 9 5}(+10)$ & $\mathbf{1 0 9 1}(-2)$ & $\mathbf{1 0 2 2}(+8)$ & $\mathbf{1 1 0 0}(-13)$ \\
$\mathrm{TR}_{\mathrm{O}} / \mathrm{CS}$ & $\mathbf{3 0 8}(+93)^{* * *}$ & $\mathbf{5 9 5}(-38)^{* *}$ & $\mathbf{2 8 7}(+65)^{* * *}$ & $\mathbf{4 7 4}(-46)^{* * *}$ \\
$\mathrm{ET}_{\mathrm{O}} / \mathrm{CS}$ & $\mathbf{6 8 7}(-28)^{* *}$ & $\mathbf{4 9 5}(+43)^{* *}$ & $\mathbf{7 3 4}(-15)^{*}$ & $\mathbf{6 2 5}(+11)$ \\
$\mathrm{F}_{\mathrm{V}} / \mathrm{F}_{\mathrm{M}}$ & $\mathbf{2 2 2}(-58)$ & $\mathbf{9 3}(+162)^{* * *}$ & $\mathbf{2 5 7}(-38)^{* *}$ & $\mathbf{1 5 9}(+53)^{*}$ \\
$\mathrm{PI}_{\mathrm{ABS}}$ & $\mathbf{0 , 7 0}(-37)^{* *}$ & $\mathbf{0 , 4 4}(+54)^{* * *}$ & $\mathbf{0 , 7 3}(-25)^{* * *}$ & $\mathbf{0 , 5 4}(+37)^{* *}$ \\
\hline
\end{tabular}

Média de 10 repetições por período. As porcentagens entre parêntesis referem-se às mudanças promovidas pela condição de luz, valores negativos indicam redução em relação à condição de exposição anterior, enquanto valores positivos indicam aumento. * $\mathrm{p}<0,05 ; * * \mathrm{p}<0,01 ; * * * \mathrm{p}<0,001$

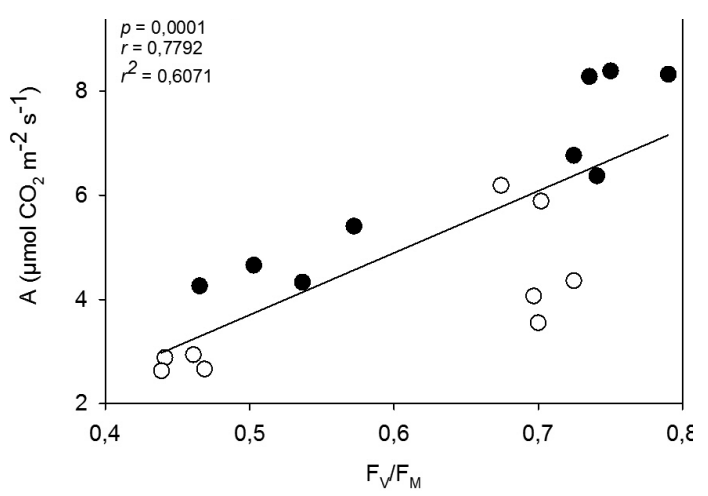

Figura 2 - Correlação entre fotossíntese e máxima eficiência fotoquímica do PSII $\left(\mathrm{F}_{\mathrm{V}} / \mathrm{F}_{\mathrm{M}}\right)(\mathrm{n}=18)$. Círculos abertos = Aniba canelilla e círculos fechados = A. rosaeodora.

Figure 2 - Correlation between photosynthesis and photochemical efficiency of PSII $\left(\mathrm{F}_{\mathrm{V}} / \mathrm{F}_{\mathrm{M}}\right)(\mathrm{n}=18)$. Open circles $=$ Aniba canelilla and closed circles $=A$. rosaeodora .

retomaram o patamar anterior à exposição em pleno sol (Tab. 2).

A magnitude das respostas na análise dos parâmetros de fluorescência da clorofila $a$ foi diferente entre as espécies durante a exposição à condição de pleno sol (Fig. 3a-f). Aniba rosaeodora apresentou taxas de captação de energia $\left(\mathrm{TR}_{\mathrm{O}} / \mathrm{CS}\right)$ e transporte de elétrons $\left(\mathrm{ET}_{\mathrm{O}} / \mathrm{CS}\right)$ cerca de 26 e $72 \%$ superiores à $A$. canelilla (Fig. $3 \mathrm{c}, \mathrm{d}$ ). Aniba rosaeodora apresentou valores de $\mathrm{F}_{\mathrm{V}} / \mathrm{F}_{\mathrm{M}}$ cerca de $11 \%$ maiores em relação à $A$. canelilla durante todo o período experimental (Fig. 3e).

Quanto à plasticidade foliar, verificou-se que Aniba canelilla e A. rosaeodora apresentaram plasticidade superior a $60 \%$ (Tab. 3). Para $A$. canelila e $A$. rosaeodora todas as variáveis com exceção de $\mathrm{ABS} / \mathrm{CS}, \mathrm{TR}_{\mathrm{O}} / \mathrm{CS}, \mathrm{F}_{\mathrm{V}} / \mathrm{F}_{\mathrm{M}}$ e $C i$ apresentaram índice de plasticidade foliar superior a 50\% (Tab. 3).

\section{Discussão}

A variação na disponibilidade de luz influenciou as respostas fotossintéticas de Aniba canelilla e $A$. rosaeodora. De modo geral, verificou-se em ambas as espécies redução da capacidade fotossintética quando as plantas foram expostas à condição de pleno sol. Em contrapartida, a submissão novamente à sombra, após tratamento no sol, promoveu maior desempenho fotoquímico e das taxas de assimilação de carbono.

A redução na capacidade fotossintética das duas espécies em pleno sol foi determinada pela queda na eficiência fotoquímica $\left(\mathrm{F}_{\mathrm{V}} / \mathrm{F}_{\mathrm{M}} \mathrm{e}\right.$ $\mathrm{PI}_{\mathrm{ABS}}$ ). Por outro lado, o aumento da capacidade fotossintética sob sombra pode ser atribuído além da recuperação de $\mathrm{F}_{\mathrm{V}} / \mathrm{F}_{\mathrm{M}}$ e $\mathrm{PI}_{\mathrm{ABS}}$, pela retomada dos 


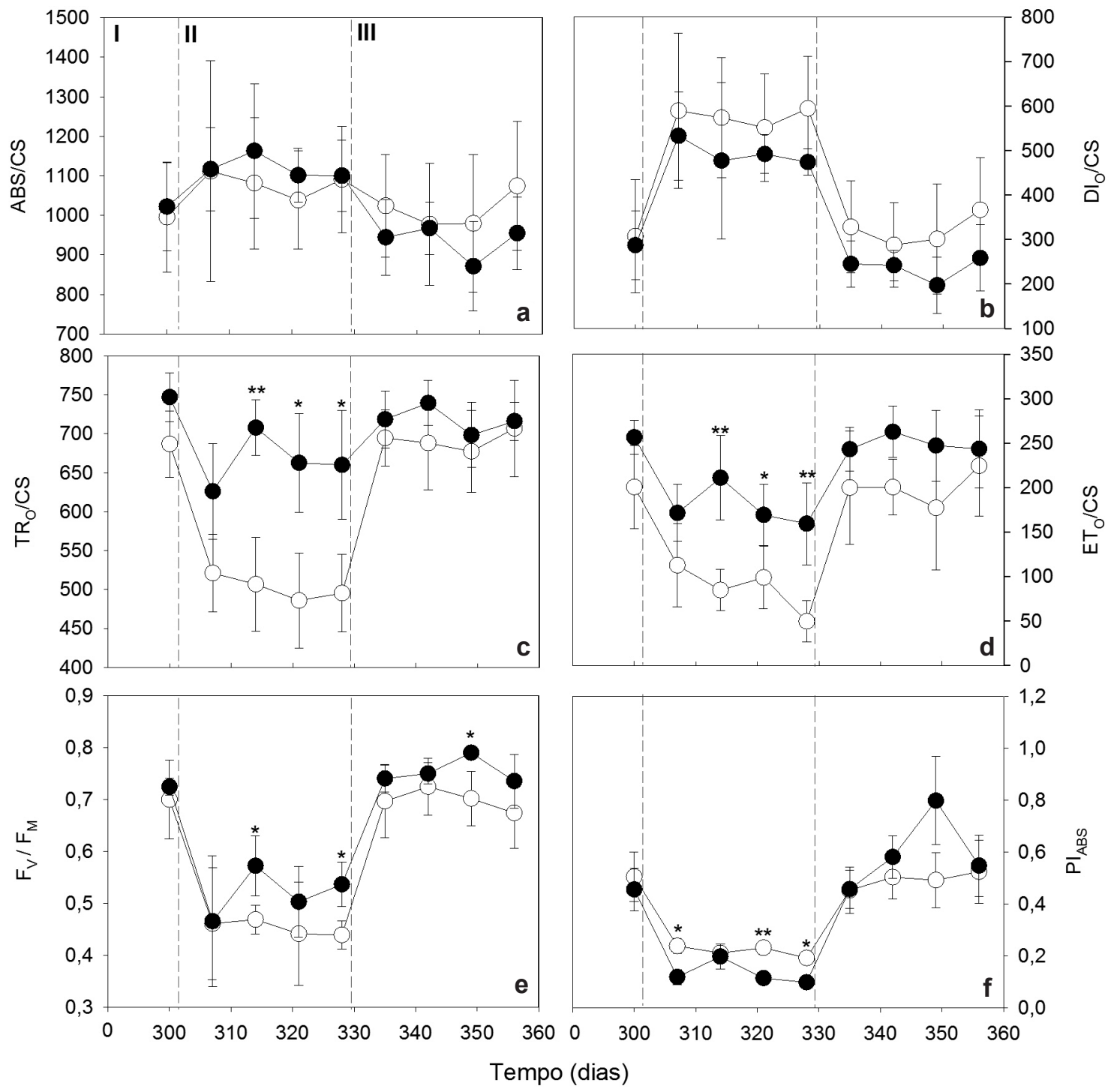

Figura 3 - Mudanças nos parâmetros de fluorescência da clorofila $a$ de $A$. canelilla $(\bigcirc)$ e $A$.rosaeodora $(\bullet)$ promovidas pela variação na disponibilidade de luz $(\mathrm{n}=10)$. As linhas pontilhadas indicam o momento em que foi realizada a intervenção no plantio. I = aclimatação; II = pleno sol; III = sombreamento. a. eficiências de absorção (ABS/CS); b. dissipação (DI $\mathrm{CS})$; c. aprisionamento $\left(\mathrm{TR}_{\mathrm{O}} / \mathrm{CS}\right)$; d. transferência $\left(\mathrm{ET}_{\mathrm{O}} / \mathrm{CS}\right)$ de energia; e. rendimento quântico máximo do PSII $\left(\mathrm{F}_{\mathrm{v}} / \mathrm{F}_{\mathrm{M}}\right)$; f. índice de desempenho na base $\mathrm{ABS}\left(\mathrm{PI}_{\mathrm{ABS}}\right)$.

Figure 3 - Changes in chlorophyll $a$ fluorescence parameters of $A$. canelilla $(\bigcirc)$ and $A$. rosaeodora $(\bullet)$ promoted by variation in light availability $(\mathrm{n}=10)$. Dotted lines indicate the time at which the intervention was performed at planting. I = acclimatization; II = full sunlight; $\mathrm{III}=$ shading. a. absorption flux per CS (ABS/CS); b. energy dissipation flux $\left(\mathrm{DI}_{\mathrm{o}} / \mathrm{CS}\right)$; c. exciton trapping flux $\left(\mathrm{TR}_{\mathrm{o}} / \mathrm{CS}\right)$; d. electron transport flux $\left(\mathrm{ET}_{\mathrm{O}} \mathrm{CS}\right)$; e. maximum quantum yield of PSII $\left(\mathrm{F}_{\mathrm{V}} / \mathrm{F}_{\mathrm{M}}\right)$; f. performance index on $\mathrm{ABS}$ base $\left(\mathrm{PI}_{\mathrm{ABS}}\right)$.

valores de condutância estomática $(g s)$. Resultados semelhantes foram verificados em plantas jovens de Aniba rosaeodora submetidas a níveis crescentes de irradiância (Gonçalves et al. 2005). Os autores observaram que a capacidade fotossintética dessa espécie foi inversamente proporcional à intensidade de irradiância, sendo $g s$ e $\mathrm{F}_{\mathrm{V}} / \mathrm{F}_{\mathrm{M}}$ as principais variáveis que contribuíram para esses resultados. $\mathrm{O}$ aumento da irradiância sob condições hídricas e nutricionais adequadas, normalmente 
Tabela 3 - Plasticidade foliar de plantas jovens de Aniba canelilla e A. rosaeodora submetidas à condições contrastantes de irradiância. Fotossíntese $(A)$, condutância estomática $(g s)$, respiração no escuro $(R d)$, transpiração $(E)$ e concentração interna de $\mathrm{CO}_{2}(\mathrm{Ci})$, índice do conteúdo de clorofilas (ICC), eficiência de absorção (ABS/CS), dissipação $\left(\mathrm{DI}_{\mathrm{O}} / \mathrm{CS}\right)$, aprisionamento $\left(\mathrm{TR}_{\mathrm{O}} / \mathrm{CS}\right)$ e transferência $\left(\mathrm{ET}_{\mathrm{O}} / \mathrm{CS}\right)$ de energia, rendimento quântico máximo do PSII $\left(\mathrm{F}_{\mathrm{V}} / \mathrm{F}_{\mathrm{M}}\right)$ e índice de desempenho na base ABS $\left(\mathrm{PI}_{\mathrm{ABS}}\right)$.

Table 3 - Leaf plasticity of young Aniba canelilla and $A$. rosaeodora plants submitted to contrasting irradiance conditions. Photosynthesis $(A)$, stomatal conductance $(g s)$, dark respiration $(R d)$, transpiration $(E)$ and internal $\mathrm{CO}_{2}$ concentration $(C i)$, chlorophyll content (ICC), absorption flux per CS (ABS/CS), energy dissipation flux ( $\left.\mathrm{DI}_{\mathrm{O}} / \mathrm{CS}\right)$, exciton trapping flux $\left(\mathrm{TR}_{\mathrm{O}} / \mathrm{CS}\right)$ and electron transport flux $\left(\mathrm{ET}_{\mathrm{o}} /\right.$ $\mathrm{CS})$, maximum quantum yield of PSII $\left(\mathrm{F}_{\mathrm{V}} / \mathrm{F}_{\mathrm{M}}\right)$ and performance index based on $\mathrm{ABS}\left(\mathrm{PI}_{\mathrm{ABS}}\right)$.

\begin{tabular}{|c|c|c|}
\hline \multirow{2}{*}{ Traço foliar } & \multicolumn{2}{|c|}{ Índice de plasticidade (\%) } \\
\hline & Aniba canelilla & Aniba rosaeodora \\
\hline$A$ & 96,67 & 74,93 \\
\hline$R d$ & 80,38 & 89,74 \\
\hline$g s$ & 97,56 & 84,11 \\
\hline$E$ & 96,00 & 74,07 \\
\hline $\mathrm{Ci}$ & 51,85 & 26,28 \\
\hline ICC & 74,04 & 81,10 \\
\hline $\mathrm{ABS} / \mathrm{CS}$ & 33,73 & 30,51 \\
\hline $\mathrm{DI}_{\mathrm{o}} / \mathrm{CS}$ & 74,38 & 63,38 \\
\hline $\mathrm{TR}_{\mathrm{O}} / \mathrm{CS}$ & 55,40 & 38,15 \\
\hline $\mathrm{ET}_{\mathrm{o}} / \mathrm{CS}$ & 87,50 & 59,95 \\
\hline $\mathrm{F}_{\mathrm{V}} / \mathrm{F}_{\mathrm{M}}$ & 62,02 & 46,72 \\
\hline $\mathrm{PI}_{\mathrm{ABS}}$ & 99,52 & 93,53 \\
\hline Plasticidade foliar média & 77,93 & 66,93 \\
\hline
\end{tabular}

promove o aumento da condutância estomática (Marenco et al. 2014). A concentração interna de $\mathrm{CO}_{2}(\mathrm{Ci})$, por sua vez, também influencia a abertura e o fechamento dos estômatos. Neste caso, há uma tendência de decréscimo da condutância estomática com o aumento da concentração interna de $\mathrm{CO}_{2}$ (Lawson et al. 2008). O aumento da concentração interna de $\mathrm{CO}_{2}(\mathrm{Ci})$ pode estar associado à redução da condutância estomática em situações na qual ocorre uma redução da assimilação de $\mathrm{CO}_{2}$ em resposta a queda na produção de trifosfato de adenosina (ATP) (Lawson et al. 2008). No presente estudo, o aumento da concentração interna de $\mathrm{CO}_{2}$ em pleno sol e posterior redução na sombra parece ter sido determinante para as respostas estomáticas de Aniba canelilla e A. rosaeodora.

$\mathrm{O}$ aumento da concentração interna de $\mathrm{CO}_{2}$ em Aniba canelilla e $A$. rosaeodora quando expostas a pleno sol pode ter ocorrido em função do processo de fotoinibição verificado neste período. $\mathrm{O}$ pior desempenho fotoquímico (menores valores de $\mathrm{F}_{\mathrm{V}} / \mathrm{F}_{\mathrm{M}}$ e $\mathrm{PI}_{\mathrm{ABS}}$ ) indica redução na produção de ATP e NADPH, podendo comprometer a assimilação de $\mathrm{CO}_{2}$ (Wong et al. 2012; Janka et al. 2015). À semelhança dos resultados alcançados neste estudo, a fotoinibição também foi considerada a principal causa da redução nas taxas fotossintéticas de $A$. rosaeodora quando exposta a elevados níveis de irradiância (Gonçalves et al. 2005) e a redução da eficiência fotoquímica também influenciou as repostas fotossintéticas de Euterpe edulis Mart. crescendo sob condições contrastantes de irradiância (sub-bosque e clareira) (Lavinsky et al. 2014).

A redução de $\mathrm{F}_{\mathrm{V}} / \mathrm{F}_{\mathrm{M}}$ e $\mathrm{PI}_{\mathrm{ABS}}$ em Aniba canelilla e $A$. rosaeodora sob pleno sol e sua posterior recuperação ao serem submetidas novamente à sombra está associada principalmente às mudanças promovidas nos parâmetros $\mathrm{DI}_{\mathrm{O}} / \mathrm{CS}$, $\mathrm{TR}_{\mathrm{O}} / \mathrm{CS}$ e $\mathrm{ET}_{\mathrm{O}} / \mathrm{CS}$. As respostas fotoquímicas de plantas submetidas à variação na disponibilidade de irradiância são determinadas em grande medida pela capacidade das espécies na regulação da dissipação não fotoquímica (Hallik et al. 2012; Janka et al. 2015). Nesse sentido, o aumento da dissipação não fotoquímica em $A$. canelilla e $A$. rosaeodora em pleno sol e redução em sombra pode ser interpretado como estratégia adotada por estas 
espécies para amenizar os fotodanos promovidos pelo excesso de irradiância.

Alterações nas concentrações foliares de pigmentos cloroplastídicos também constituem estratégias para alcançar a melhor eficiência fotoquímica (Schöttler \& Tóth 2014). Neste sentido, a redução na concentração de pigmentos (ICC) em pleno sol e o posterior aumento em sombra também pode ser entendido como estratégia de fotoaclimatação. Sob alta irradiância, há um desequilíbrio entre a síntese e degradação dos pigmentos cloroplastídicos, com predominância da degradação por foto-oxidação, enquanto que na sombra, onde a luz é um recurso limitante, as plantas podem aumentar o conteúdo de pigmentos para captar mais energia (Hallik et al. 2012; Silva et al. 2015). É importante destacar que as respostas dependem da espécie e das condições hídricas e nutricionais das plantas (Malavasi \& Malavasi 2001; Rodrigues \& Gonçalves 2014).

Em relação à plasticidade, constatou-se que Aniba canelilla e $A$. rosaeodora exibem estratégias fisiológicas que as permitem suportar variações na disponibilidade de luz. Neste caso, observou-se que a plasticidade fisiológica de $A$. canelilla e $A$. rosaeodora parece ser conferida, em curto prazo, pela capacidade dessas espécies regularem a dissipação não fotoquímica $\left(\mathrm{DI}_{\mathrm{O}} /\right.$ CS), a condutância estomática e o conteúdo de pigmentos cloroplastídicos. À semelhança do que foi observado neste estudo, também se verificou que outras espécies não pioneiras (Cedrela odorata L., Manilkara salzmannii (A.DC.) H.J. Lam.e Cariniana estrellensis (Raddi) Kuntze) também exibiram plasticidade quando expostas à condições contrastantes de irradiância, neste caso a plasticidade foi associada principalmente ao ajuste do conteúdo de pigmentos cloroplastídicos e da taxa de assimilação líquida de $\mathrm{CO}_{2}$ (Gaburro et al. 2015).

Diante das informações aqui apresentadas no que tange ao desempenho da fotossíntese de plantas jovens de Anibae, nós verificamos que a variação na disponibilidade de irradiância influenciou o desempenho fotossintético de Aniba canelilla e $A$. rosaeodora. As espécies reduziram a capacidade fotossintética durante a exposição a pleno sol, porém se recuperaram ao serem submetidas novamente à sombra. Este comportamento sugere que ambas as espécies apresentam plasticidade fisiológica quando submetidas a condições contrastantes de irradiância, o que pode subsidiar a recomendação de $A$. canelilla e $A$. rosaeodora para a composição de sistema silviculturais de enriquecimento em ambientes com frequentes variações na disponibilidade de luz desde que o plantio seja gradativo observando a maior demanda de luz para $A$. rosaeodora. Portanto, concluímos que nossos resultados suportam a hipótese de serem estas espécies adequadas para o enriquecimento gradativo de florestas secundárias na Amazônia.

\section{Agradecimentos}

Os autores desta pesquisa agradecem ao Instituto Nacional de Pesquisas da Amazônia (MCTI-INPA), o suporte logístico; ao Laboratório de Fisiologia e Bioquímica Vegetal, a infraestrutura disponibilizada para realização desta pesquisa; à FAPEAM e à CAPES, as concessões das bolsas (Mestrado a Michell Richard Blind, e Doutorado a Carlos Eduardo Moura da Silva). O Dr. José Francisco de Carvalho Gonçalves agradece ao CNPq, a concessão da bolsa de Produtividade em pesquisa.

\section{Referências}

Amusant N, Digeon A, Descroix L, Bruneau O, Bezard $\mathrm{V}$ \& Beauchène $\mathrm{J}$ (2015) Planting rosewood for the sustainable essential oil production: Influence of surrounding forest and seed provenance on tree growth and essential oil yield. Bois et Forêst des Tropiques 304: 57-65.

Amusant N, Beauchène J, Digeon A \& Chaix G (2016) Essential oil yield in rosewood (Aniba rosaeodora Ducke): initial application of rapid prediction by near infrared spectroscopy based on wood spectra. Journal of Near Infrared Spectroscopy 24: 507-515.

Atroch EMAC (2008) Efeitos da disponibilidade hídrica e de diferentes condições de irradiância sobre o crescimento, características fotossintéticas e o acúmulo de óleos voláteis em plantas de Aniba rosaeodora Ducke e Aniba canelilla (Kunth) Mez (Lauraceae). Tese de Doutorado. Instituto Nacional de Pesquisas da Amazônia, Manaus. 180p.

Doucet J-L, Dainou K, Ligot G, Ouédraogo D-Y, Bourland N \& Ward SE (2016) Enrichment of Central African logged forests with highvalue tree species: testing a new approach to regenerating degraded forests. International Journal of Biodiversity Science 12: 83-95.

Ferraz SFB, Ferraz KMPMB, Cassiano CC, Brancalion PHS, Luz DTA, Azevedo TN, Tambosi LR \& Metzger JP (2014) How good are tropical forest patches for ecosystem services provisioning? Landscape Ecology 29: 187-200.

Gaburro TA, Zanetti LV, Gama VN, Milanez CRD \& Cuzzuol GRF (2015) Physiological variables related to photosynthesis are more plastic than the 
morphological and biochemistry in non-pioneer tropical trees under contrasting irradiance. Brazilian Journal of Botany 38: 39-49.

Giongo JL, Vaucher RA, Da Silva AS, Oliveira CB, Mattos CB, Baldissera MD, Sagrillo MR, Monteiro SG, Custódio DL, Matos MS, Sampaio PT, Teixeira HF, Koester LS \& Veiga Junior VF (2017) Trypanocidal activity of the compounds present in Aniba canelilla oil against Trypanosoma evansi and its effects on viability of lymphocytes. Microbial Pathogenesis 103: 13-18.

Goldstein G, Santiago LS, Campanello PI, Avalos G, Zhang Y-J \& Villagra M (2016) Facing shortage or excessive light: How tropical and subtropical trees adjust their photosynthetic behavior and life history traits to a dynamic forest environment. Tropical Tree Physiology 6: 319-336.

Gonçalves JFC, Barreto DCS, Santos Jr. UM, Fernandes AV, Sampaio PTB \& Buckeridge MS (2005) Growth, photosynthesis and stress indicators in young rosewood plants (Aniba rosaeodora Ducke) under different light intensities. Brazilian Journal Plant Physiology 17: 325-334.

Goncalves JFC, Santos Jr. UM \& Silva EA (2008) Evaluation of a portable chlorophyll meter to estimate concentrations in leaves of tropical wood species from Amazonian forest. Hoehnea 35: 185-188.

Hallik L, Niinemets Ü \& Kull O (2012) Photosynthetic acclimation to light in woody and herbaceous species: a comparison of leaf structure, pigment content and chlorophyll fluorescence characteristics measured in the field. Plant Biology 14: 88-89.

Instituto Nacional de Meteorologia (2017) Portal do Instituto Nacional de Meteorologia. Disponível em <http://www.inmet.gov.br/portal $>$. Acesso em 10 fevereiro 2017.

Instituto Nacional de Pesquisas Espaciais (2017) TerraClass 2012. Disponível em <http://www. inpe.br/cra/projetospesquisas/terraclass2010.php $>$. Acesso em 9 janeiro 2017.

International Union for Conservation of Nature (2017) The IUCN red list threatened species. Version 2014.3. Disponível em <http://www.iucnredlist. org $>$. Acesso em 9 fevereiro 2017.

Janka E, Körner O, Rosenqvist E \& Ottosen C-O (2015) Using the quantum yields of photosystem II and the rate of net photosynthesis to monitor high irradiance and temperature stress in chrysanthemum (Dendranthema grandiflora). Plant Physiology and Biochemistry 90: 14-22.

Karsten RJ, Jovanovic M, Meilby H, Perales E \& Reynel C (2013). Regeneration in canopy gaps of tierra-firme forest in the Peruvian Amazon: comparing reduced impact logging and natural unmanaged forest. Forest Ecology and Management 310: 663-671.

Köppen W (1948) Climatologia: un estúdio de los climas de la tierra. Fondo de Cultura Econômica, México. 479p.
Lavinsky AO, Gomes FP, Mielke MS \& França S (2014) Photosynthetic acclimation in shade-developed leaves of Euterpe edulis Mart (Arecaceae) after long-term exposure to high light. Photosynthetica 52: 351-357.

Lawson T, Lefebvre S, Baker NR, Morison JIL \& Rainers CA (2008) Reductions in mesophyll and guard cell photosynthesis impact on the control of stomatal responses to light and $\mathrm{CO}_{2}$. Journal of Experimental Botany 59: 3609-3619.

Malavasi UC \& Malavasi MM (2001) Leaf characteristics and chlorophyll concentration of Schyzolobium parahybum and Hymenaea stilbocarpa seedlings grown in different light regimes. Tree Physiology 21: 701-703.

Marenco RA, Nascimento HCS \& Magalhães NS (2014) Stomatal conductance in Amazonian tree saplings in response to variations in the physical environment. Photosynthetica 52: 1-8.

Nava DB, Monteiro EA, Correia MC, Araújo MR, Sampaio RRL \& Campos GS (1998) Sócioeconomia do município de Presidente Figueiredo, Amazonas. Disponível em < http://www.cprm.gov.br/publique/ media/socioeco.pdf>. Acesso em 10 maio 2017.

Parry C, Blonquist Jr JM \& Bugbee B (2014) In situ measurement of leaf chlorophyll concentration: analysis of the optical/absolute relationship. Plant and Cell Environment 37: 2508-2520.

Pinheiro KAO, Carvalho JOP, Quanz B, Francez LMB \& Schwartz G (2007) Fitossociologia de uma área de preservação permanente no Leste da Amazônia: indicação de espécies para recuperação de áreas alteradas. Floresta 37: 175-187.

Rappaport D \& Montagnini F (2014) Tree species growth under a rubber (Hevea brasiliensis) plantation: native restoration via enrichment planting in southern Bahia, Brazil. New Forest 245: 715-732.

Reis LP, Carvalho JOP, Reis PCM, Gomes JM, Ruschel AR \& Silva MG (2014) Crescimento de mudas de Parkia gigantocarpa Ducke, em um sistema de enriquecimento em clareiras após a colheita de madeira. Ciência Florestal 24: 431-436.

Ribeiro RV, Souza GM, Oliveira RF \& Machado EC (2005) Photosynthetic responses of tropical tree species from different successional groups under contrasting irradiance conditions. Revista Brasileira de Botânica 28: 149-161.

Rodrigues JVFC \& Gonçalves JFC (2014) Leaf gas exchange, photon capture and light harvest in Aldina heterophylla along a vegetation gradient in the Amazon Rainforest. American Journal of Plant Sciences 5: 1477-1488.

Sampaio PTB, Barbosa AP, Vieira G, Spironello WR, Ferraz IDK, Camargo JLC \& Quisen RC (2003) Silvicultura do pau-rosa (Aniba rosaeodora Ducke). In: Higuchi N, Santos J, Sampaio PTB, Marenco 
RA, Ferraz J, Sales PC, Saito S \& Matsumoto S (eds.) Projeto Jacaranda-Fase II. INPA, Manaus. Pp 179-189.

Schöttler MA \& Tóth SZ (2014) Photosynthetic complex stoichiometry dynamics in higher plants: environmental acclimation and photosynthetic flux control. Plant Science 5: 1-15.

Silva AF, Oliveira RV, Santos NRL \& de Paula A (2003) Composição florística e grupos ecofisiológicos das espécies de um trecho de floresta semidecídua submontana da fazenda São Geraldo. Árvore 27: 311-319.

Silva CEM, Gonçalves JFC \& Feldpausch TR (2008) Water-use efficiency of tree species following calcium and phosphorus application on an abandoned pasture, central Amazonia, Brazil. Environmental and Experimental Botany 64: 189-195.

Silva CEM, Gonçalves JFC \& Alves EG (2011) Photosynthetic traits and water use of tree species growing on abandoned pasture in different periods of precipitation in Amazonia. Photosynthetica 49: 246-252.

Silva CS, Seider WD \& Lior N (2015) Exergy efficiency of plant photosynthesis. Chemical Engineering Science 130: 151-171.

Strasser RJ, Srivastava A \& Govindjee (1995) Polyphasic chlorophyll $a$ fluorescence transient in plants and cyanobacteria. Photochemistry and Photobiology 61: 32-42.

Tanaka A \& Vieira G (2006) Autoecologia das espécies florestais em regime de plantio de enriquecimento em linha na floresta primária da Amazônia Central. Acta Amazonica 36: 193-204.

Valladares F, Wright JS, Lasso E, Kitajima K \& Pearcy RW (2000) Plastic phenotypic responses to light of 16 congeneric shrubs from a Panamanian rainforest. Ecology 81: 1925-1936.

Wandelli EV \& Fearnside PM (2015) Secondary vegetation in central Amazonia: land use history effects on aboveground biomass. Forest Ecology and Management 347: 140-148.

Wong SL, Chen CW, Huang HW \& Weng JH (2012) Using combined measurements of gas exchange and chlorophyll fluorescence to investigate the photosynthetic light responses of plant species adapted to different light regimes. Photosynthetica 50: 206-214.

Zama MY, Bovolenta YR, Carvalho ES, Rodrigues DR, Araujo CG, Sorace MAF \& Luz DG (2012) Florística e síndromes de dispersão de espécies arbustivo-arbóreas no Parque Estadual Mata São Francisco, PR, Brasil. Hoehnea 39: 369-378. 